\title{
Bayesian statistical inference of the loglogistic model with interval-censored lifetime data
}

\begin{abstract}
The properties of Palm Oil (PO) and Coconut Oil (CO) offer the potential for transformers Interval-censored data arise when a failure time say, $\mathrm{T}$ cannot be observed directly but can only be determined to lie in an interval obtained from a series of inspection times. The frequentist approach for analysing interval-censored data has been developed for some time now. It is very common due to unavailability of software in the field of biological, medical and reliability studies to simplify the interval censoring structure of the data into that of a more standard right censoring situation by imputing the midpoints of the censoring intervals. In this research paper, we apply the Bayesian approach by employing Lindley's 1980, and Tierney and Kadane 1986 numerical approximation procedures when the survival data under consideration are interval-censored. The Bayesian approach to interval-censored data has barely been discussed in literature. The essence of this study is to explore and promote the Bayesian methods when the survival data been analysed are is interval-censored. We have considered only a parametric approach by assuming that the survival data follow a loglogistic distribution model. We illustrate the proposed methods with two real data sets. A simulation study is also carried out to compare the performances of the methods.
\end{abstract}

Keyword: Interval censoring; Bayesian inference; Maximum likelihood; Gamma priors; Squared error; LINEX loss functions 